\title{
Frecuencia de Pseudomonas aeruginosa en bolsas periodontales de pacientes con Periodontitis Crónica
}

\author{
Carlos Rafael I nvernizzi-Mendoza ${ }^{1}$, Humberto Corbeta ${ }^{1}$ \\ ${ }^{1}$ Universidad Autónoma de Asunción, Facultad de Odontología, Cátedra de Periodoncia I y II. \\ Asunción, Paraguay
}

Cómo referenciar este artículo/ How to reference this article:

\begin{abstract}
I nvernizzi-Mendoza CR, Corbeta H. Frecuencia de Pseudomonasa eruginosa en bolsas periodontales de pacientes con Periodontitis Crónica.Mem. Inst. Investig. Cienc. Salud. 2020; 18(3): 73-78
\end{abstract}

\section{RE S U ME N}

La periodontitis crónica es una patología caracterizada por la destrucción de los tejidos de soporte del diente. Existe evidencia científica de la presencia en bolsas periodontales de Pseudomonas aeruginosa, bacteria que altera la microbiota subgingival. Ha sido asociada al fracaso en el tratamiento de la periodontitis y podría constituir un riesgo para la salud general de los pacientes. El objetivo de esta investigación fue determinar la frecuencia de $P$. aeruginosa en bolsas periodontales de pacientes con periodontitis crónica que acudieron a la cátedra de Periodoncia de la Universidad Autónoma de Asunción. El estudio fue observacional descriptivo de corte transversal, para el mismo fueron seleccionados pacientes con periodontitis crónica que cumplían con los criterios de inclusión. Las piezas dentariasseleccionadas para la toma de muestras fueron aisladas con rollos de algodón estéril, y una vez removida la placa bacteriana supragingival, se retiró la placa subgingival de las bolsas periodontales por medio de curetas de Gracey y se introdujeron en tubos de ensayo que contenían medio de Stuart, para luego llevar las muestras hasta el laboratorio de microbiología para su análisis. Del total de 14 muestras, solo una dio positivo a $\mathrm{P}$. aeruginosa representando el 7,14\%. El microrganismo aislado resultó resistente a los antibióticos utilizados como coadyuvantes en el tratamiento de la periodontitis crónica,al igual que en otros trabajos encontrados en la literatura internacional. La presencia de estas bacterias dificulta el control de la enfermedad periodontal y podría significar un riesgo para desarrollar infecciones oportunistas, especialmente en los pacientes inmunodeprimidos.

Palabras clave: Periodontitis, microbiología periodontal, microbiota subgingival, Pseudomonas aeruginosa

\section{Frequency of Pseudomonas aeruginosa in periodontal pockets of patients with Chronic Periodontitis}

\footnotetext{
ABSTRACT

Chronic periodontitis is a pathology characterized by the destruction of tooth supporting tissues. There is scientific evidence of the presence in periodontal pockets of Pseudomonas aeruginosa, a bacterium that alters the subgingival microbiota and that has been associated with failure to treat periodontitis and could constitute a risk to patient general health. The objective of this research was to determine the frequency of $\mathrm{P}$. aeruginosa in periodontal pockets of patients with chronic periodontitis who attend the Chair of Periodontology at the Autonomous University of Asunción. Descriptive, cross-sectional observational study, in which patients with chronic

Fecha de recepción: setiembre 2020. Fecha de aceptación: octubre 2020

* Autor correspondiente: Dr. Carlos Rafael I nvernizzi-Mendoza. Docente Investigador de Odontología. Cátedra de Periodoncia I y II. Universidad Autónoma de Asunción. Email:carlosinvernizzi@hotmail.com Co-autor: Dr. Humberto Corbeta. Docente Cátedra de Periodoncia I y II. Universidad Autónoma de Asunción. Email: hcorbeta@gmail.com.
} 
periodontitis who met the inclusion criteria were selected. The pieces selected for sampling were isolated with sterile cotton rolls, and once the supragingival bacterial plaque had been removed, the subgingival plaque was removed from the periodontal pockets by means of Gracey curettes and placed in test tubes containing Stuart medium, then the samples were taken to the microbiology lab for analysis. Of the total of 14 samples, only one was positive for P. aeruginosa, representing $7.14 \%$. The isolated microorganism was resistant to the antibiotics used as adjuvants in the treatment of chronic periodontitis, as in other studies found in the international literature. The presence of these bacteria makes it difficult to control periodontal disease and could pose a risk for developing opportunistic infections, especially in immunosuppressed patients.

Keywords: Periodontitis, periodontal microbiology, subgingival microbiota, Pseudomonas aeruginosa.

\section{NTRODUCCI ÓN}

La periodontitis crónica es una patología infecciosa caracterizada por la destrucción de los tejidos de soporte del diente, y es el resultado de la respuesta inflamatoria local y sistémica del huésped susceptible, motivado por la liberación de productos bacterianos específicos de la placa dental ${ }^{(1-4)}$. Esta enfermedad está ampliamente distribuida en el mundo, afectando aproximadamente al $48 \%$ de la población adulta, en una fase avanzada causa perdida de las piezas dentarias, afectando la estética y funcionalidad, con un impacto en la calidad de vida de los pacientes ${ }^{(5-7)}$.

Los microrganismos asociados con esta patología ya han sido plenamente identificados, son bacilos Gram negativos anaerobios estrictos y entre los más importantes podemos mencionar a Porphyromonas gingivalis, Aggregatibacter actinomycetemcomitans, Tannerella forsythia y Treponema denticola ${ }^{(4,8,9)}$.

A pesar de esto, existe evidencia científica de la presencia en la cavidad bucal, específicamente en las bolsas periodontales, de otras bacterias potencialmente patógenas como Pseudomonas aeruginosa, que podrían constituir un riesgo para la salud general de los pacientes, aunque su frecuencia en bolsas periodontales es muy variable en todo el mundo ${ }^{(5,8-10)}$. Al principio se pensó que la presencia de este tipo de microorganismos se daba de manera transitoria en la cavidad bucal, o que tal vez era producto de una contaminación a la hora de la toma de muestras, pero con las últimas investigaciones se ha comprobado que la cavidad bucal definitivamente puede constituirse en un reservorio de este y otros microrganismos patógenos ${ }^{(11-17)}$.

Actualmente se han descubierto cepas multirresistentes de $P$. aeruginosa que representan un peligro para la salud general de los pacientes. Presenta entre sus principales factores de virulencia, la elevada resistencia a los antibióticos, y entre ellos a los utilizados como coadyuvantes del tratamiento periodontal. Otro factor de virulencia importante de esta bacteria es la capacidad de producir y secretar potentes toxinas y enzimas extracelulares; además, suprime la proliferación de linfocitos y es bastante invasiva, capaz de adherirse y formar biofilms en tejidos y superficies abióticas ${ }^{(16-19)}$.

La importancia de la presencia de esta bacteria en las bolsas periodontales radica en que altera la microbiota subgingival y ha sido asociada al fracaso en el tratamiento de la periodontitis, tanto mecánico, por la dificultad de erradicar estas bacterias de bolsas profundas, y al tratamiento antimicrobiano, por su elevada resistencia a los fármacos más utilizados en el tratamiento ${ }^{(20,21)}$.

Ardila ${ }^{(22)}$ halló que la presencia de este tipo de microrganismo y el hábito de fumar están correlacionados de manera positiva, también observó correlaciones positivas en cuanto a la presencia de estos microrganismos y la gravedad de la destrucción periodontal, sugiriendo que existe peores condiciones periodontales en los pacientes que presentan este tipo de microrganismo. Varios autores sugieren que factores como, la respuesta del huésped, la composición de la microbiota, el acceso a la atención odontológica, hábitos de higiene oral y el tabaquismo pueden ayudar a explicar las correlaciones entre la expresión clínica de la periodontitis y la presencia de bacterias inusuales en las bolsas periodontales ${ }^{(23-27)}$. 
Souza Goncalves ${ }^{(14)}$ realizó un estudio comparativo entre pacientes con salud sistémica y pacientes infectados con el Virus de Inmunodeficiencia Humana $(\mathrm{VIH})$, encontrando que es mayor la frecuencia de P. aeruginosa en pacientes con este virus. Aún si se encuentran en bajos niveles, esta bacteria puede implicar un peligro para la salud de este tipo de pacientes inmunocomprometidos. Los estudios de Souza demuestran que los pacientes con $\mathrm{VIH}$ y diagnosticados con periodontitis crónica tienen una prevalencia menor de $\mathrm{P}$. aeruginosa que los que tienen $\mathrm{VIH}$ y salud periodontal ${ }^{(14)}$.

Da Silva-Boghossian ${ }^{(13)}$ demostró que Aggregatibacter actinomycetemcomitans, A. baumannii y las bacterias del complejo rojo (Tannerella forsythia, Porphyromonas gingivalis y Treponema denticola) pueden asociarse con P. aeruginosa en la microbiota subgingival y aumentar significativamente la probabilidad de desarrollar una periodontitis agresiva, esto indica que existiría una sinergia entre P. aeruginosa y A. actinomycetemcomitans, lo que aumenta bastante el riesgo de la progresión a una enfermedad más destructiva. Las interacciones que se producen entre P. aeruginosa y las bacterias periodonto patógenas podrían ayudar a la capacidad que tiene $P$. aeruginosa de invadir células epiteliales y diseminarse de manera sistémica ${ }^{(28)}$.

En Paraguay existe evidencia sobre la gran resistencia a antibióticos por especies de P. aeruginosa, especialmente relacionadas con el uso inapropiado de antibióticos ${ }^{(29)}$ y las Infecciones Asociadas a la Atención Sanitaria (IAAS) ${ }^{(30)}$ pero no existen estudios referentes a la presencia de este microrganismo en bolsas periodontales, por lo que se considera importante investigar dicha situación. El objetivo de esta investigación fue determinar la frecuencia de P. aeruginosa en bolsas periodontales de pacientes diagnosticados con periodontitis crónica que acudieron a la cátedra de Periodoncia de la carrera de Odontología de la Universidad Autónoma de Asunción durante el año 2016.

\section{MATERI ALES Y MÉTODOS}

Estudio observacional descriptivo de corte transversal, en donde se seleccionaron pacientes diagnosticados con periodontitis crónica que asistían a la Cátedra de Periodoncia de la Carrera de Odontología de la Facultad de Ciencias de la Salud de la Universidad Autónoma de Asunción durante el año 2016.

Los pacientes cumplieron con los criterios de inclusión, que fueron los siguientes:

- Pacientes de sexo masculino y femenino, sin distinción de edad, diagnosticados con periodontitis crónica que no recibieron terapia local o sistémica con antibióticos por lo menos 3 meses antes de la toma de muestra

- Pacientes que no hayan sido tratados periodontalmente por lo menos 6 meses antes de la toma de muestra

- Pacientes con por lo menos 6 dientes naturales en boca

- Pacientes que una vez explicados los objetivos de esta investigación dieron su consentimiento por escrito para participar de la misma

Para la toma de muestras se seleccionó una pieza dentaria de cada sectante con diagnóstico de periodontitis crónica, luego se aisló la zona con rollos de algodón estéril, y una vez removida la placa bacteriana supragingival, se retiró la placa subgingival de las bolsas periodontales por medio de curetasde Gracey (Hu-Friedy) y se introdujeron en tubos de ensayo que contenía medio de Stuart, para luego llevar las muestras hasta el laboratorio de microbiología para su posterior análisis. Las muestras fueron cultivadas en agar MacConkey en estufa de cultivo a $37^{\circ}$ durante 48 horas. Una vez revisadas las placas con crecimiento, se realizaron pruebas bioquímicas para la identificación de género y especie. Posteriormente se realizó antibiograma para observar la resistencia y sensibilidad a los fármacos. Los datos obtenidos fueron asentados en planillas Excel y utilizados exclusivamente para el presente trabajo. Se respetaron todos los principios éticos durante el desarrollo de la investigación. 


\section{RESULTADOS}

Se evaluaron muestras de placa subgingival de 14 pacientes con periodontitis crónica, 7 de sexo masculino y 7 femenino. Del total de 14 muestras, solo una dio positivo a Pseudomonas aeruginosa representando el 7,14\%. Se realizó la sensibilidad a antibióticos in vitro por el método de difusión en disco, demostrando que el microrganismo era resistente a los principales fármacos coadyuvantes en el tratamiento periodontal, como la Amoxicilina, Amoxicilina asociada a Acido clavulánico y Azitromicina. Entre los fármacos efectivos contra este microorganismo se encontró a la ciprofloxacina, la cual podría ser útil en el tratamiento (Ver Tabla 1).

Tabla 1. Sensibilidad y Resistencia de P. aeruginosa antes distintos fármacos.

\begin{tabular}{|c|c|c|}
\hline Microrganismo & Sensible a: & Resistente a: \\
\hline $\begin{array}{l}\text { Pseudomonas } \\
\text { aeruginosa }\end{array}$ & $\begin{array}{l}\text { Ceftazidima, Piperacilina, } \\
\text { Piperacilina/Tazobactam, } \\
\text { Ciprofloxacina, Gentamicina, } \\
\text { Amikacina }\end{array}$ & $\begin{array}{l}\text { Amoxicilina, Ampicilina, } \\
\text { Amoxicilina/ Ac. } \\
\text { clavulánico, Azitromicina }\end{array}$ \\
\hline
\end{tabular}

\section{DISCUSIÓN}

La frecuencia de P. aeruginosa en el presente estudio fue del 7,14\%, cifra bastante similar a los trabajos de Betancourth ${ }^{(9)}$, que encontró una frecuencia del $7 \%$ en pacientes con periodontitis crónica y $1,6 \%$ en periodontitis agresiva en el Valle del Cauca, Colombia. Ardila ${ }^{(22)}$ en Antioquia-Colombia reportó una frecuencia del 5,26\%; Silva ${ }^{(10)}$ en Santiago-Chile encontró una frecuencia de $4 \%$ y Barbosa ${ }^{(31)}$ en Sao PauloBrasil halló en total un $10 \%$ de P. aeruginosa en pacientes con Periodontitis.

Resultados superiores a este estudio, fueron los presentados por Botero(11) en CaliColombia, que encontró un $15,79 \%$ en pacientes con periodontitis crónica y Souto ${ }^{(8)}$ reportó una prevalencia del $52,2 \%$ en el biofilmsubgingival de pacientes con periodontitis crónica y $11,4 \%$ en pacientes con salud periodontal, además menciona que no hubo diferencias significativas en cuanto al sexo, aunque si mayor frecuencia en pacientes fumadores. La presencia elevada de este tipo de microrganismo también puede estar relacionada con el consumo de alimentos y agua contaminada, e incluso con una inadecuada higiene personal ${ }^{(32)}$.

A pesar de estos resultados, existen estudios en donde la frecuencia de $P$. aeruginosa es mínima o nula, como en el estudio de Slots ${ }^{(32)}$, que encontró $0 \%$ de Pseudomonas en pacientes con periodontitis crónica avanzada en República Dominicana. Slots sugirió que la frecuencia de este tipo de bacterias aumenta a medida que incrementa la edad, debido a una acumulación de estos microorganismos con el paso de los años, y menciona que los hombres pueden presentar mayor frecuencia debido a que realizan menos consultas odontológicas que las mujeres ${ }^{(33)}$.

En cuanto a la resistencia a antibióticos de $P$. aeruginosa, en este estudio se encontró que es resistente a los antibióticos utilizados como coadyuvantes en el tratamiento de la periodontitis crónica. Otros autores como Barbosa ${ }^{(31)}$, también reportan que el $87,5 \%$ de $P$. aeruginosa aislado de bolsas periodontales era resistente a la Amoxicilina/Ac. Clavulánico y así como en esta investigación, encontró a la Ciprofloxacina como un antimicrobiano eficaz para eliminar a esta bacteria.

Por otro lado, la Ciprofloxacina al ser un antibiótico de amplio espectro puede suprimir gran parte de la microbiota normal, lo que podría ser contraproducente en la cavidad oral. Investigaciones demuestran que en comunidades microbianas muy organizadas las Pseudomonas pueden interactuar con otras bacterias produciendo intercambios frecuentes de genes de resistencia a múltiples fármacos ${ }^{(34)}$.

Una de las limitaciones del presente estudio se encuentra en el tamaño de la muestra, esto debido al costo de los estudios microbiológicos y la necesidad de una estricta selección de los pacientes, aun así, se recomienda elevar el número de muestras para estudiar a profundidad la importancia y el rol de este microrganismo en la periodontitis crónica. 
Del total de 14 pacientes, solo uno dio positivo a $\mathrm{P}$. aeruginosa, que representa el $7,14 \%$ de la muestra. El microrganismo aislado es resistente a la mayoría delos antibióticos utilizados como coadyuvantes en el tratamiento de la periodontitis crónica, por lo que su presencia dificultaría el control de la enfermedad periodontal y podría significar un riesgo para desarrollar infecciones oportunistas, especialmente en los pacientes inmunodeprimidos.

\section{Conflicto de Interés}

Los autores declaran no tener conflictos de interés.

\section{REFERENCI AS BIBLI OGRÁFI CAS}

1. Berglundh $T$, Donati $M$. Aspects of adaptive host response in periodontitis.

J Clin Periodontol 2005; 32: 87-107.

2. Paster BJ, Olsen I, Aas JA, Dewhirst FE. The breadth of bacterial diversity in the human periodontal pocket and other oral sites. Periodontol 2000. 2006; 42: 80-87.

3. Kinane DF, Attstrom R. Advances in the pathogenesis of periodontitis. Group B consensus report of the Fifth European Workshop in Periodontology. J Clin Periodontol 2005; 32:130-131.

4. Socransky SS, Haffajje AD, Cugini MA, Smith CM, Kent RL Jr. Microbial complexes in subgingival plaque. J Clin Periodontol 1998; 25: 134-44.

5. Ardila CM, Arbeláez MI, Guzmán IC. Perfil microbiológico subgingival de pacientes con periodontitis crónica en una población de Colombia. Avances en Periodoncia. 2012 Abr; 24 (1): 47-53.

6. Albandar JM. Epidemiology and risk factors of periodontal diseases. Dent Clin North Am 2005; 49: 517-32.

7. World Health Organization. Oral health surveys. Basic methods. Geneva: World Health Organization; 2013.

8. Souto R, Silva-Boghossian C, Colombo A. Prevalence of Pseudomonas aeruginosa and Acinetobacter spp. in subgingival biofilm and saliva of subjects with chronic periodontal infection. Brazilian Journal of Microbiology 2014. 45 (2): 495-501

9. Betancourth $M$, Arce $R$, Botero J, Jaramillo A, Cruz C, Contreras A. Microorganismos inusuales en surcos $y$ bolsas periodontales. Colomb Med 2006; 37: 6-14

10. Silva SN, Gamonal J, Gajardo M. Ocurrencia de bacilos Gram negativos no fastidiosos y levaduras en la microbiotasubgingival en periodontitis. Revista Odontológica Mexicana 2006; 10 (3): 119-25

11. Botero JE, Contreras A, Lafaurie G, Jaramillo A, Betancourt $M$, Arce RM.
Occurrence of periodontopathic and superinfecting bacteria in chronic and aggressive periodontitis subjects in a Colombian population. J Periodontol 2007; 78:696-704.

12. Colombo AP, Teles RP, Torres MC, Souto R, Rosalem WJ, Mendes MC et al. Subgingival microbiota of Brazilian subjects with untreated chronic periodontitis. J Periodontol 2002; 73: 360-9

13. Da Silva-Boghossian CM, Souto R, Luiz $\mathrm{RR}$, Colombo APV. Association of red complex, A. actinomycetemcomitans and non-oral bacteria with periodontal diaseases. Arch Oral Biol 2011; 56:899906

14. Goncalves L de S, Souto R, Colombo AP. Detection of Helicobacter pylori, Enterococcus faecalis, and Pseudomonas aeruginosa in the subgingival biofilm of HIV-infected subjects undergoing HAART with chronic periodontitis. Eur J ClinMicrobiol Infect Dis 2009; 28: 1335-42.

15. Souto R, Colombo AP. Detection of Helicobacter pylori by polymerase chain reaction in the subgingival biofilm and saliva of non-dyspeptic periodontal patients. J Periodontol 2008; 79:97103.

16. Slots J, Feik D, Rams TE. Prevalence and antimicrobial susceptibility of Enterobacteriacea, Psedomonadaceaea and Acinetobacter in human periodontitis. Oral Microbiol Immunol 1990; 5: 149-54.

17. Slots J, Rams TE, Litsgarten MA. Yeasts, enteric rods and pseudomonads in the subgingival flora of severe adult periodontitis. Oral Microb Immunol 1988; 3:47-52.

18. Pihl MLEC, Schimidtchen A, Svensater G, Davies JR. Effects of clinical isolates of Pseudomonas aeruginosa on Staphylococcus epidermidis biofilm formation. FEMS Immunol Med Microbiol 2010; 59: 504-12. 
19. Smith RS, Iglewski BH. P. aeruginosa quorum-sensing systems and virulence. Curr Opin Microbiol 2010; 6:56-60

20. Colombo AP, Boches SK, Cotton SL, Goodson J M, Kent R, Haffajee AD et al. Comparisons of subgingival microbial profiles of refractory periodontitis, severe periodontitis, and periodontal health using the human oral microbe identification microarray. J Periodontol 2009; 80: 1421-32.

21. Colombo AP, Haffajee AD, Dewhirst FE, Paster BJ, Smith CM, Cugini $M$ A et al. Clinical and microbiological features of refractory periodontitis subjects. J Clin Periodontol 1998, 25: 169-80.

22. Ardila Medina CM, Alzate Vega J, Guzmán Zuluaga IC. Correlación de bacilos entéricos gram-negativos con parámetros clínicos y demográficos de pacientes con periodontitis crónica. Av Period on Implantol. 2014; 26 (2): 7782.

23. Mager DL, Haffajee AD, Socransky SS. Effects of periodontitis and smoking on the microbiota of oral mucous membranes and saliva in systemically healthy subjects. J Clin Periodontol 2003; 30: 1031-7.

24. Haffajee AD, Socransky SS. Relationship of cigarette smoking to the subgingival microbiota. J Clin Periodontol 2001; 28:377-88.

25. Haffajee AD, Socransky SS. Relationship of cigarette smoking to attachment level profiles. J Clin Periodontol 2001; 28: 283-95.

26. Wara-aswapati N, Pitiphat W, Chanchaimongkon $L$, Taweechaisupapong S, Boch JA, Ishikawa I. Red bacterial complex is associated with the severity of chronic periodontitis in a Thai population. Oral Dis 2009; 15:354-9.

27. Torrungruang K, Bandhaya $P$, Likittanasombat K, Grittayaphong C. Relationship between the presence of certain bacterial pathogens and periodontal status of urban Thai adults. J Periodontol 2009;80: 122-9
28. Pan $Y$, Teng $D$, Burke AC, Haase EM, Scannapieco FA. Oral bacteria modulate invasion and induction of apoptosis in HEp-2 cells by Pseudomonas aeruginosa. Microb Pathog. 2018; 46, 73-9.

29. Rodríguez AJ, Samaniego DR, Soskin A, et al. Comparative study of antimicrobial resistance of Pseudomonas aeruginosa strains isolated from patients of Caracas and Asunción in a 4-year-period. Chemotherapy. 2002; 48(4):164-7.

30. Pedrozo Torres ME, Vázquez FA, Holt N, Cabello MA, Samudio M, Baruja D, et al. Brote en simultáneo de Pseudomona aeruginosa y Klebsiella pneumoniae BLEE en una Unidad de Cuidados Intensivos Neonatal de Asunción, Paraguay. Mem. Inst. Investig. Cienc. Salud. 2019; 17(1): 59-68.

31. Barbosa FCB, Mayer MPA, Saba-Chujfi E, Cai S. Subgingival occurrence and antimicrobial susceptibility of enteric rods and pseudomonads from Brazilian periodontitis patients. Oral Microbiol Immunol 2001: 16: 306-10.

32. Slots J, Rams TE, Feik D, Taveras HD, Gillespie GM. Subgingival microflora of advanced periodontitis in the Dominican Republic. J Periodontol 1991; 62: 5437.

33. Slots J, Feik D, Rams TE. Age and sex relationships of superinfecting microorganisms in periodontitis patients. Oral Microbiol Immunol 1990; 5: 305-8.

34. Hansen SK, Haagensen JA, Gjermansen $M$, Jorgensen T. M, Tolker-Nielsen $T$, Molin S. Characterization of a Pseudomonas putida rough variant evolved in a mixed species biofilm with Acinetobacter sp. strain C6. J Bacteriol. 2007; 189:4432-93. 